\title{
Appearance-focused Internet Use and the Thin-beauty Ideal
}

\author{
Tyne Stanley ${ }^{1}$, James Barnes ${ }^{1}$, Emma Short ${ }^{1}$ \\ ${ }^{1}$ National Centre for Cyberstalking Research, University of Bedfordshire, UK \\ Correspondence: Emma Short, National Centre for Cyberstalking Research, University of Bedfordshire, UK
}

Received: June 5, 2015 Accepted: July 2, 2015 Online Published: July 24, 2015

doi:10.11114/smc.v3i2.971 URL: http://dx.doi.org/10.11114/smc.v3i2.971

\begin{abstract}
Websites featuring appearance-focused content are a medium for constant appearance comparisons, addiction and pressure to meet existing beauty-ideals. This study investigated a sample of 264 males and females who use appearance-focused websites, with a view to determining the relationship with appearance dissatisfaction and self-worth. Data collection involved posting links to online questionnaires on popular social networking sites. Internet appearance exposure was found to correlate with a greater drive for thinness in females, suggesting that exposure to the thin-ideal body images presented online reinforces women's desire to achieve the cultural expectations of body shape and weight. Furthermore the study highlighted that internet addiction was associated with a drive for thinness and low self-esteem in both males and females, with differences exhibited in regards to body-esteem. These findings emphasise the need for pathological internet use to be incorporated into media literacy programs and to encourage a critical stance toward current beauty standards.
\end{abstract}

Keywords: social networking, body image, internet addiction, thin beauty ideal, appearance-focused internet use

\section{Introduction}

\subsection{Body Dissatisfaction}

Extensive dissatisfaction with body shape and weight has frequently been observed in research sampling women and adolescent girls, with body concerns being described as normative discontent (Rodin, Silberstein, \& Striegel-Moore, 1984). Body dissatisfaction is described as a negative subjective evaluation of the weight and shape of one's body, and has been identified as a robust risk factor for eating disorders (Wade \& Tiggemann, 2013). Longitudinal studies have indicated that body dissatisfaction is one of the most significant precursors to negative emotional states and unhealthy body-related behaviours; therefore, researchers have expressed the importance of developing a greater understanding of the risk factors that lead to an overall dissatisfaction with one's body (Dittmar, 2009).

Prominent theories postulated to account for the development of body dissatisfaction typically emphasise the influential role of developmental and sociocultural factors (Heinberg, 1996). Developmental theories posit that childhood and adolescent development factors, such as teasing about appearance and the timing of the onset of puberty, are central to the formation of body image disturbance in adulthood (Heinberg, 1996). Sociocultural theories argue that the pressure to conform to current thin-beauty ideals comes from a variety of sociocultural influences, the most pervasive being the media, and is recognised as a widely accepted explanation for the aforementioned phenomena (Tiggemann \& Slater, 2013). Others contend that our exposure to the sociocultural beauty standards presented in the media significantly increases the risk of body image disturbance and eating pathology (Heinberg \& Thompson, 1995)

\subsection{The Thin-ideal}

Thin-ideal internalisation, the extent to which individuals endorse the socially defined ideals of attractiveness, has been identified as a mediator in the relationship between media exposure and dissatisfaction with shape and weight (Dittmar \& Howard, 2004). Research has shown that exposure to the unrealistic beauty standards portrayed in the media contributes significantly to the development of eating disorders such as anorexia-nervosa and bulimia (Spettigue \& Henderson, 2004; Tiggemann, 2003). In a study which examined the relationship between thin-ideal internalisation, body dissatisfaction, and symptoms of eating disorders within a sample of adolescent girls, it was found that pressure from family and peers, in combination with the acceptance of social standards related to appearance, contributed significantly to the development of eating pathology (Rukavina \& Pokrajac-Bulian, 2006). Such findings indicate that the perception and acceptance of social standards regarding shape and weight has a profound influence on eating habits 
and behaviours.

\subsection{Media Influences}

An extensive amount of research exists to support the notion that exposure to the thin-ideal body images presented within magazines and/or television advertisements is associated with body image concerns (Grabe, Ward, \& Hyde, 2008). However, although adolescents and younger adults are especially avid adopters, social networking is growing in popularity for older adults. The Office for National Statistics reports that in 2012, $48 \%$ of adults and $87 \%$ of $16-24$ year olds use social networking sites such as Facebook and Twitter to communicate online. A recent growth has been documented in individuals accessing social networks via mobile devices, with percentages reported as $63 \%$ of adults and $72 \%$ of 16-24 year olds; culminating in a culture of continuous exposure to media images (ONS, 2012).

Websites amalgamated with appearance-focused content are commonplace and are often targeted at young girls. They frequently carry health and beauty, clothing, pro-anorexia and celebrity content. (Subrahmanyam \& Greenfield, 2008; Tiggemann \& Miller, 2010) contend that the developmental issues associated with adolescence are being played out online, with social networking sites often being their primary source of contact with offline friends. Although reviews have shown that these sites can offer a sense of social connectedness and well-being, they also provide a medium for constant appearance comparison; thereby adding pressure to meet existing beauty-ideals (Tiggemann \& Miller, 2010).

\subsection{Appearance-Focused Internet Use}

Tiggemann and Miller (2010) investigated the relationship between internet-appearance exposure and body image concerns in a sample of 156 adolescent girls. Questionnaires were distributed that contained measures of the thin-ideal internalisation, physical appearance comparison, weight satisfaction, drive for thinness and specific internet usage. As predicted, internet appearance exposure was significantly associated with weight dissatisfaction and a drive for thinness. Additionally, those who spent more time on social networking sites (e.g. Facebook) experienced higher levels of weight dissatisfaction, a drive for thinness and internalisation of the thin-ideal. In contrast, time spent on websites such as Google and YouTube showed no association. Other researchers argue that intense forms of appearance conversations occur on social networks, which is a predictor of poor body image (Clark \& Tiggemann, 2006). Such findings suggest that a type of engagement with particular sites is inherently negative for body image, not purely modal use of the internet.

Due to advances in technology, a large proportion of individuals are adopting the use of the internet for work and social purposes. Despite its advantages, the internet has provided a medium that is proving addictive for some (Siomos $\&$ Angelopoulos, 2008). Recent empirical findings have begun to identify a potential relationship between excessive internet use and body image dissatisfaction, which has created a niche area for further study (Ceyhan, Ceyhan, \& Kurtyilmaz, 2012). Internet Addiction Disorder (IAD) is a term used to describe excessive internet use which results in academic, social and occupational impairment; however, it is not yet recognised as a clinical disorder (Young, 1998b). The condition consists of various subtypes characterised by various compulsive internet activities, such as gambling, pornography use, shopping and gaming (Weinstein \& Lejoyeux, 2010). Internet use is considered a problem when an individual exhibits symptoms such as losing themselves online, spending longer online than intended, neglecting real-life relationships as a result of focusing on internet relationships, reliance on its use for stress relief and denial of time spent online (Song, LaRose, Eastin, \& Lin, 2004).

Weinstein and Lejoyeux postulated a variety of reasons to explain excessive use of the internet, including that of a coping mechanism against underlying psychological developing issues, and as a forum for expanding social networks (and consequently enhancing meaningful relationships, confidence and social support) (Weinstein \& Lejoyeux, 2010). 'Self-Presentational Theory' contends that the internet provides a safe place for socially anxious individuals to interact and a medium for control over their image (Rodgers, Melioli, Laconi, Bui, \& Chabrol, 2013). Indeed, a connection has been identified between shyness and internet addiction (Chak \& Leung, 2004). Suler proposed the concept of the 'Online Disinhibition Effect' as a possible explanation for internet addiction, explaining that the dissociative anonymity and invisibility of cyberspace allows everyday internet users to feel less restrained, and thus, more able to express themselves (Suler, 2004).

Young explains that individuals who possess maladaptive cognitions, such as low self-esteem and self-worth, are at high risk of becoming addicted to the internet, as its capacity for anonymous interactions allows them to overcome perceived inadequacies (Young, 1998b). An unhealthy use of the internet can increase a person's susceptibility to various social, academic and professional difficulties (Ceyhan, 2008). Subsequent studies have found that internet addiction is associated with lower levels of self-esteem, depression, life satisfaction, and mental ill health; thus hindering an individual's coping skills and ability to adapt (Yang \& Tung, 2007; Young, 1998b). Since it has been discovered that pathological internet use has a detrimental impact on social and emotional functioning, various studies have been conducted to examine psychological dependency on the internet (Ceyhan, et al., 2012). However, the role of satisfaction 
with physical appearance as a predictor of problematic internet use has received little empirical attention.

Koronczai and colleagues contend that dissatisfaction with bodily appearance often leads to individuals avoiding personal contact, and adopting an increased use of the internet to partake in social interactions (Koronczai et al., 2013). When exploring this relationship, Koronczai et al. found that satisfaction with body appearance had a significant effect on self-esteem, anxiety and problematic internet use amongst males and females, with the body satisfaction-problematic internet use relationship being mediated via the self-esteem-depression path. It appears that low self-esteem and poor self-image play a significant role in individuals immersing themselves online to avoid face-to-face contact. However, participants included in the study varied dramatically in terms of age (14 to 34 years) and it is possible that younger participants may have experienced different levels of self-esteem and body dissatisfaction compared to those over the age of 30. Tiggemann and Lynch found that although levels of body dissatisfaction remained stable across a sample of women ranging from 20 to 84 years, factors such as self-objectification, appearance anxiety, and habitual body monitoring significantly decreased with age (Tiggemann \& Lynch, 2001).

\subsection{Internet Addiction and Body Image}

Rodgers and others further explored the association between internet addiction and body image concerns through the distribution of online questionnaires to 392 young adults (Rodgers, et al., 2013). Measures included time spent online, internet addiction symptoms, disordered eating, and body image avoidance. Internet addiction was found to be associated with body image avoidance in both genders, and, when controlling for the body-mass index (BMI), it was a significant predictor of disordered eating amongst women. A moderate association was identified between body image avoidance, disordered eating, and time spent on communication websites, suggesting that our perception of how others evaluate our appearance in combination with the desire to control self-presentation, may contribute to becoming addicted to the internet. However, a prominent issue with the aforementioned studies is their inability to determine the temporal relationships between the variables. It has also been suggested that the association between internet addiction and body dissatisfaction takes an indirect form, explaining that individuals who are dissatisfied with their body often suffer from deficient social support and problem solving skills, which exposes individuals to feelings of depression, and, consequently, increases the likelihood of carrying out problematic behaviours online (Ceyhan, et al., 2012).

A prominent limitation regarding the majority of the aforementioned studies is that their samples revolve primarily around adolescent girls; however, a growing body of research has indicated that men also experience anxiety when it comes to their bodies (Burlew \& Shurts, 2013). In a similar way to women, dissatisfaction with body shape and weight in the male population is believed to be triggered by exposure to images of the traditional ideal male masculine body depicted in the media (Morris \& Katzman, 2003). For instance, Smolak and Stein identified a relationship between the number of idealised male bodies portrayed in the media and the prevalence of body dissatisfaction and weight disorders in men (Smolak \& Stein, 2006). Body image concerns amongst men are thought to be fuelled by exposure to idealised images of women with men experiencing higher levels of self-consciousness regarding their bodies after reading magazines that featured images of scantily-clad thin women, concluding that this exposure creates pressure for men to obtain similar idealised physiques (Aubrey \& Taylor, 2009).

\subsection{Gender Differences in Body Dissatisfaction}

Research has highlighted that although both men and women experience dissatisfaction with their bodies, women generally experience lower levels of body satisfaction in comparison to men (Algars et al., 2009). Furthermore, whilst women exhibit a desire to lose weight, men focus on increasing muscle mass (Petrie, Greenleaf, \& Martin, 2010). Men are more likely to report that they are lighter in comparison to their actual weight and are less likely to rate their bodies as unattractive; hence demonstrating key differences in the way that men and women respond to questions about their bodies (Rasmussen, Eriksson, \& Nordquist, 2007) When exploring the underlying causes of body dissatisfaction competitiveness and pressure to have a better body were identified as key contributors in men (Blashill, 2011), whereas the perceived pressure to meet societal standards of the perfect body appeared a crucial determinate in women (Knauss, Paxton, \& Alsaker, 2007). Therefore, whilst external pressures appear to be the primary drive behind the male body image, internal pressures are more influential to the construction of the female body image.

Franzoi and Shields recognised that body-esteem (an important dimension of self-esteem that involves self-evaluations of one's body or appearance) is a multidimensional construct that differs for men and women (Franzoi \& Shields, 1984). Previously, measures such as the Body-Cathexis Scale (Secord \& Jourard, 1953) assumed that body-esteem could be expressed as a single score constructed from an individual's preferential responses to a list of body items; however, studies conducted after its development questioned the assumption of unidimensionality (Fisher, 1964). Whilst developing a new measure of body-esteem, Franzoi and Shields conducted a factor analysis which revealed that the major body-esteem dimensions differed for men and women. For men, body-esteem concerns are centred on their physical condition, upper body strength, and physical attractiveness, whereas for women it is primarily related to sexual 
attractiveness, physical condition, and weight (Franzoi \& Shields, 1984). Further evidence of gender differences in regards to body image concerns comes from Fernandez and Pritchard, who found that although media models were the primary predictor of a drive for thinness in both men and women, the secondary predictor differed (social pressures for women, internalisation for men) (Fernandez \& Pritchard, 2012). Such findings highlight the importance of examining the impact of media sources on men and women separately to decipher gender differences.

\subsection{Research Aims}

Literature reviews have led to the conclusion that very little research exists that specifically examine the impact that constant exposure to appearance-focused internet content has on overall body image. Furthermore, past research has focused solely on body dissatisfaction amongst adolescent females. Adults are increasingly partaking in the use of social networks and are thereby being bombarded with images exemplifying the importance of looking youthful. Pressure to maintain these standards when experiencing the physiological changes associated with aging, may result in long-lasting body dissatisfaction (Marshall, Lengyel, \& Utioh, 2012). Research has indicated that exposure to images featuring the ideal male body has a significant negative impact on body dissatisfaction in men (Blond, 2008). Such findings highlight the necessity for research incorporating the areas of body dissatisfaction that have greater relevance to the adult and male populations. The primary objective of the current study was to evaluate the contribution of internet appearance exposure and internet addiction in predicting the levels of body dissatisfaction, drive for thinness, body-esteem and self-esteem; within a more varied sample in terms of age and gender.

\section{Method}

\subsection{Design}

A correlational research design was employed to examine the predictive value of internet use in the acquisition of body image concerns. The variables included internet appearance exposure, internet addiction, drive for thinness, body dissatisfaction, low self-esteem and body-esteem.

\subsection{Participants}

Participants were recruited by posting links to an online Qualtrics survey on social networking sites including Facebook and Twitter. As well as voluntary participation, the snowball effect was also acquired, as participants frequently passed on the details of the study to others. Four hundred and seven participants volunteered to complete the online survey; of those, 264 completed the entire survey (65\%): 191 were female (72\%) and 73 were male (28\%). 81\% were Caucasian, $7 \%$ were Mixed, $8 \%$ were Asian, $2 \%$ were African Caribbean, and 2\% identified themselves as Other. Ages ranged from 18 to 64: 145 were 18-24 (55\%), 69 were 25-34 (26\%), 20 were 35-44 (8\%), 22 were 45-54 (8\%), and 8 were 55-64 (3\%).

\subsection{Demographic Information}

Participants were asked to provide details regarding their basic demographics, which included their age, gender, ethnic group identification, height, and weight (see Table 1.)

\subsection{Materials}

\subsubsection{Internet Appearance Exposure}

A modified version of the Internet Appearance Exposure Scale (Tiggemann \& Miller, 2010) was used to measure the extent to which people use websites that feature appearance-focused content. The websites mentioned in question six were modified to represent the most popular websites of UK audiences (Ebizmba, 2013b). Similarly, the social networking sites (SNS) mentioned in question five were modified to represent the most popular SNS of UK audiences (Ebizmba, 2013a). A number of questions regarding internet usage were featured, such as how long they spend on the internet during the week and weekend (none, 30 minutes or less, one hour, 2-3 hours, four hours or more), where they learn about websites (e.g. friends, search engines, other websites) and which SNS they use (e.g. Facebook, Twitter). Participants were asked which of the 19 options (e.g. emails, searching for images, fashion websites) they use the internet for, to which they were required to rank in order the three activities they spend the most time on. The final question detailed a list of 10 popular websites compiled from current internet ratings (Ebizmba, 2013b), with the option to add a further two websites. Internet sites were then rated by three independent raters on appearance focus: zero (no emphasis), one (some emphasis), and two (large emphasis), with a total internet appearance exposure score being calculated by multiplying the rating with the frequency with which the site was viewed and summing across sites.

\subsubsection{Internet Addiction}

Internet addiction was assessed using the Internet Addiction Test (Young, 1998a). This scale assesses the degree to which an individual's internet use affects their daily routine, social life, productivity, sleeping patterns and feelings. It consists of 20 items (e.g., "How often do you find yourself anticipating when you will go on-line again?") with which 
respondents rate their agreement on a five-point scale (rarely to always). Higher scores indicate greater problems with internet use, with possible scores ranging from 20 to 100. In the present sample, the scale had high internal reliability ( $\alpha$ $=.91)$

\subsubsection{Drive for Thinness}

Drive for thinness was assessed using the Drive for Thinness subscale of the Eating Disorder Inventory-3 (EDI-3) (Garner, 2004). This subscale assesses preoccupation with dieting, weight, and an extreme desire to be thinner. It consists of seven items (e.g., "I am terrified of gaining weight") rated on a 0-4 point scoring system (never to always). Higher scores indicate a greater drive for thinness, with possible scores ranging from 0 to 28 . In the present sample, the subscale demonstrated high internal reliability $(\alpha=.93)$.

\subsubsection{Body Dissatisfaction}

Body dissatisfaction was assessed by the Body Dissatisfaction subscale of the EDI-3 (Garner, 2004). This subscale assesses discontentment with the overall shape and size of different regions of the body (i.e. stomach, hips, and thighs). It consists of 10 items (e.g., "I feel satisfied with the shape of my body") rated on a 0-4 point scoring system (never to always). Higher scores indicate greater levels of body dissatisfaction, with possible scores ranging from 0 to 40 .- In the present sample, the subscale had high internal reliability $(\alpha=.92)$.

\subsubsection{Low Self-esteem}

Self-esteem was assessed by the Low Self-Esteem subscale of the EDI-3 (Garner, 2004). This subscale assesses the basic concept of negative self-evaluation. It consists of six items (e.g., "I feel ineffective as a person"), five of which measure feelings of inadequacy and lack of personal worth, with the final item measuring the perception of being unable to achieve personal standards; rated on a 0-4 point scoring system (never to always). Higher scores reflect lower levels of self-esteem, with possible scores ranging from 0 to 24 . In the present sample, the subscale had high internal reliability $(\alpha=.93)$.

\subsubsection{The Body-Esteem Scale}

The Body-Esteem Scale (BES) is a factorially derived measure used to assess the different dimensions of body satisfaction in men and women (Franzoi \& Shields, 1984). The original BES features a list of 35 body parts and functions (e.g. appetite, weight, and physical stamina) to which respondents indicate their feelings towards each part of their own body on a 5-point scale (strong negative feelings to strong positive feelings). In this study, two items (item number three, nose, and item number 31, sex activities, were discounted in order to reduce the number of questions. For women, three subscales were provided to measure sexual attractiveness, weight concern, and physical condition, whilst for men, the subscales measured physical attractiveness, upper body strength, and physical condition. Lower scores indicate poorer body-esteem, with possible scores ranging from 33 to 165. In the present sample, the shortened scale demonstrated adequate internal consistency $(\alpha=.96)$, with alpha coefficients for individual subscales ranging from .87 to .96 .

\subsection{Procedure}

The study was approved by the local ethics committee. Links to the online questionnaire were posted on various websites, including Facebook and Twitter. The post contained a brief outline of what the study entailed and declared that only individuals over the age of 18 were allowed to participate. Participants were shown a consenting information sheet that they were required to read before consenting to take part which made it clear that participation was voluntary and that they had the right to withdraw their answers at any time, either during the study by exiting the web browser, or afterwards by contacting the researchers. A debrief sheet was also provided the contact details of a variety of local and national support groups.

\section{Results}

To explore the link between the internet and body image measures, a series of correlational analyses were conducted. For the variables that demonstrated a significant association, various linear regressions were performed to assess the contribution of internet appearance exposure and internet addiction to predicting body dissatisfaction, drive for thinness, body-esteem, and low self-esteem.

\subsection{Gender Differences in EDI-3 Scores}

Initially, tests were performed to assess whether males and females differed in their levels of drive for thinness, body dissatisfaction, and low self-esteem. As the Body-Esteem Scale features different subscales for men and women, an exploration of gender differences was not required for this measure. From this data, it was concluded that females are 
statistically higher in DT $(\mathrm{U}=4652.00, \mathrm{p}<0.001)$, BD $(\mathrm{U}=3732.00, \mathrm{p}<0.001)$, and LSE $(\mathrm{U}=4702.00, \mathrm{p}<0.001)$. Consequently, data analysis was conducted separately for males and females.

\subsection{Descriptive Statistics}

Descriptive data provides information about internet appearance exposure, internet addiction, and body image measures (see Table 1). Both females and males indicated that their modal internet use during the week and weekend was more than four hours per day. For females, the most frequent use of the internet was for email $(n=183)$, accessing social networking sites $(n=175)$, shopping $(n=152)$ and watching videos $(n=141)$. For males, frequent use was for email and watching videos $(n=69)$, social networking sites $(n=60)$ and news/current affairs websites $(n=58)$. When asked to explicitly rank the categories in terms of time, females reported spending the most time on social networking sites $(n=149)$, emails ( $n=95)$, and homework (academic or work-related; $n=92)$; whereas men reported spending the most time on social networking sites $(n=48)$, news/current affairs websites $(n=31)$, and watching videos $(n=29)$.

Of the social networking sites, the most popular among both males and females were Facebook and Twitter, with $94 \%$ of females and $81 \%$ of males using Facebook, and $38 \%$ of females and $42 \%$ of males using Twitter. The majority of participants found out about websites through search engines (females $82 \%$; males $89 \%$ ), and friends (females and males 75\%). From a list of specific websites (see Table 2), both males and females reported spending the most time on Facebook, with females spending an average of 77 minutes per day and males 73 minutes per day. This was followed by YouTube (females 44 minutes per day; males 52 minutes per day), Google (females 40 minutes per day; males 36 minutes per day), and Twitter (females 35 minutes per day; males 31 minutes per day). Websites reported under "other" more than twice included Reddit, Tumblr, Instagram and Etsy. Participants $\mathrm{N}=52(19.77 \%)$ met the IAT's criteria for internet addiction, defined as moderate to severe levels of internet addiction (Young, 1998a) including 35 women and 17 men, with 222 participants (166 female, 56 male) meeting the criteria for mild internet addiction. Internet addiction scores ranged from 7 to 87 , including a mean score of 37.41 .

Table 1. Means and standard deviations for internet and body image measures

\begin{tabular}{|c|c|c|}
\hline & Mean (SD) & No of Participants \\
\hline \multicolumn{3}{|l|}{ Internet Appearance Exposure } \\
\hline Males & $28.03(6.87)$ & 73 \\
\hline Females & $27.89(6.22)$ & 191 \\
\hline \multicolumn{3}{|l|}{ Internet Addiction } \\
\hline Males & $37.84(13.23)$ & 73 \\
\hline Females & $37.25(13.05)$ & 191 \\
\hline \multicolumn{3}{|l|}{ BMI } \\
\hline Males & $25.73(5.28)$ & 72 \\
\hline Females & $24.32(6.15)$ & 190 \\
\hline \multicolumn{3}{|l|}{ Drive for Thinness } \\
\hline Males & $4.92(5.15)$ & 73 \\
\hline Females & $9.93(8.68)$ & 191 \\
\hline \multicolumn{3}{|l|}{ Body Dissatisfaction } \\
\hline Males & $9.96(7.10)$ & 73 \\
\hline Females & $18.62(11.12)$ & 191 \\
\hline \multicolumn{3}{|l|}{ Low Self-Esteem } \\
\hline Males & $3.75(4.64)$ & 72 \\
\hline Females & $6.41(5.53)$ & 191 \\
\hline \multicolumn{3}{|l|}{ Body-esteem } \\
\hline Physical Attractiveness (Male) & $35.49(6.33)$ & 73 \\
\hline Sexual Attractiveness (Female) & $35.74(8.36)$ & 190 \\
\hline Upper Body Strength (Male) & $31.03(7.30)$ & 73 \\
\hline Weight Concern (Female) & $26.79(10.39)$ & 190 \\
\hline Physical Condition (Male) & $44.36(9.99)$ & 73 \\
\hline Physical Condition (Female) & $26.25(7.38)$ & 190 \\
\hline
\end{tabular}

Females: Internet appearance exposure; $n=191$, range=6-39. Internet addiction; $n=191$, range=7-87. BMI; $n=190$, range $=14.33-52.34$. EDI-3; $n=191$, DT range $=0-28$, BD range $=0-40$, LSE range $=0-20$. Body-esteem Scale; $n=190$, SA range $=11-55$, WC range $=10-50, \mathrm{PC}$ range $=9-45$. Males: Internet appearance exposure; $n=73$, range $=0-45$. Internet addiction; $n=73$, range $=20-66$. BMI; $n=72$, range $=17.63-47.18$. EDI-3; $n=73$, DT range $=0-25$, BD range $=0-32$, LSE $n$ $=72$ range $=0-20$. Body-esteem Scale $n=73$, PA range $=21-50$, UBS range $=14-45$, PC range $=18-64$ :

Table 2. Means and standard deviations for website duration of visit in minutes (per day)

\begin{tabular}{lll}
\hline Website & Mean & SD \\
\hline Google & & \\
\hline
\end{tabular}




\begin{tabular}{lll}
\hline Males & $35.53(31.76)$ & 31.76 \\
Females & $39.89(42.99)$ & 42.99 \\
Facebook & $72.67(66.39)$ & 66.39 \\
Males & $77.24(66.95)$ & 66.95 \\
Females & & \\
Yahoo & $7.17(10.57)$ & 10.57 \\
Males & $9.87(16.62)$ & 16.62 \\
Females & & \\
YouTube & $52.45(68.09)$ & 68.09 \\
Males & $43.56(61.17)$ & 61.17 \\
Females & & \\
Wikipedia & $27.13(48.18)$ & 48.18 \\
Males & $17.89(18.82)$ & 18.82 \\
Females & & \\
MSN & $3.68(8.31)$ & 8.31 \\
Males & $9.80(30.83)$ & 30.83 \\
Females & & \\
Amazon & $11.40(9.88)$ & 9.88 \\
Males & $14.64(10.93)$ & 10.93 \\
Females & & \\
EBay & $10.90(19.48)$ & 19.48 \\
Males & $14.22(22.89)$ & 22.89 \\
Females & & \\
Twitter & $31.21(71.13)$ & 71.13 \\
Males & $34.71(85.34)$ & 85.34 \\
Females & & \\
Bing & $6.25(14.86)$ & 14.86 \\
Males & .39 & 1.62 \\
Females & & \\
\hline 51 range & & \\
\hline
\end{tabular}

Females; Google $(\mathrm{n}=151)$ range $=1-200$, Facebook $(\mathrm{n}=152)$ range $=2-360$, Yahoo $(\mathrm{n}=54)$ range $=0-90$, YouTube $(\mathrm{n}=121)$ range $=2-360$, Wikipedia $(n=104)$ range $=0-120, \operatorname{MSN}(n=44)$, range $=0-200$, Amazon $(n=101)$, range $=0-50$, eBay $(n=81)$ range $=0-180$, Twitter $(n=73)$, range $=0-700$, Bing $(n=33)$ range $=0-8$. Males; Google $(n=57)$ range $=0-120$, Facebook $(n=54)$ range $=0-360$, Yahoo $(n=24)$ range $=0-30$, YouTube $(n=51)$ range $=0-300$, Wikipedia $(n=47)$ range $=0-300$, MSN $(n=19)$, range $=0-30$, Amazon $(n=43)$, range $=0-35$, eBay $(n=29)$ range $=0-100$, Twitter $(n=35)$, range $=0-400$, Bing $(n=20)$ range $=0-60$.

\subsubsection{Internet Appearance Exposure \& Body Image Measures}

Correlations were conducted to detect associations between internet appearance exposure and the three subscales of the EDI-3. For females, internet appearance exposure was revealed to have a positive correlation to a drive for thinness $(\mathrm{r}=.12, \mathrm{n}=191, \mathrm{p}<0.05)$. However, no other body image variables demonstrated a significant difference. For males, internet appearance exposure was revealed to be non-significant for all body image variables ( $>0.05$ ), which was predicted for the drive for thinness ( $r=.08, \mathrm{n}=73, \mathrm{p}>0.05)$.

\subsubsection{Internet Addiction \& Body Image Measures}

Correlations were conducted to detect associations between internet addiction and the six dimensions of body dissatisfaction in males and females. For women, internet addiction was revealed to be negatively correlated with sexual attractiveness $(\mathrm{p}=-.15, \mathrm{n}=190, \mathrm{p}<0.05)$ and physical condition $(\mathrm{r}=-.26, \mathrm{n}=190, \mathrm{p}<0.001)$. Internet addiction demonstrated a non-significant relationship with weight concern $(r=-.08, n=190, p>0.05)$. For men, internet addiction was revealed to be negatively correlated with physical attractiveness $(\mathrm{r}=-.20, \mathrm{n}=73, \mathrm{p}<0.05)$, upper body strength $(\mathrm{r}=-.35, \mathrm{n}=73, \mathrm{p}<0.01)$, and physical condition $(\mathrm{r}=-.20, \mathrm{n}=73, \mathrm{p}<0.05)$.

Correlations were conducted to examine associations between internet addiction and the three subscales of the EDI-3. For females, internet addiction was revealed to be positively correlated with a drive for thinness $(\mathrm{r}=.15, \mathrm{n}=191, \mathrm{p}<0.05)$, body dissatisfaction ( $\mathrm{r}=.13, \mathrm{n}=191, \mathrm{p}<0.05)$, and low self-esteem $(\mathrm{r}=.34, \mathrm{n}=191, \mathrm{p}<0.001)$. For males, internet addiction was revealed to be significantly positively correlated with a drive for thinness $(r=.21, n=73, p<0.05)$ and low self-esteem $(\mathrm{r}=.42, \mathrm{n}=72, \mathrm{p}<0.001)$. However, internet addiction revealed a non-significant relationship with body dissatisfaction $(\mathrm{r}=.02, \mathrm{n}=73, \mathrm{p}>0.05)$.

\subsection{Regression Analysis}

Multiple regression texts have suggested that, as a rule of thumb, subject to variable ratios of 20:1 are adequate for developing prediction models, the current sample appears sufficient enough to ensure stable coefficients (Tabachnick \& Fidell, 1989). 


\subsubsection{Internet Appearance Exposure, Internet Addiction \& Drive for Thinness}

Internet appearance exposure only demonstrated a statistical correlation with a drive for thinness in females, therefore, internet addiction and internet appearance exposure were used in a standard multiple regression analysis to predict the female drive for thinness. Preliminary analyses were conducted to ensure assumptions were not violated. The prediction model was statistically significant, $\mathrm{F}(2,190)=3.17, \mathrm{p}<0.05$, and accounted for approximately $3.3 \%$ of the variance in the drive for thinness $(\mathrm{R} 2=0.033$, Adjusted $\mathrm{R} 2=0.022)$. Examining the unique contribution of individual predictors revealed neither internet appearance exposure nor internet addiction varied significantly in relation to predicting the levels of a drive for thinness.

\subsubsection{Internet Addiction \& Eating Disorder Inventory-3 Scores}

Several linear regressions were conducted to assess whether internet addiction was a significant predictor of the scores on the EDI-3. Linear regressions were performed for the female participants first. For the drive for thinness, the prediction model was statistically significant, $[\mathrm{F}(1,190)=4.19, \mathrm{p}<0.05]$, with IA accounting for approximately $2.2 \%$ of the variance of DT $(\mathrm{R} 2=0.022$, Adjusted $\mathrm{R} 2=0.017)$. For low self-esteem, the prediction model was statistically significant, $[\mathrm{F}(1,190)=23.71, \mathrm{p}<0.001]$, with IA accounting for approximately $11.1 \%$ of the variance of LSE $(\mathrm{R} 2=$ 0.111 , Adjusted R2 $=0.107$ ). However, for body dissatisfaction the prediction model was not statistically significant ( $>0.05$ ).

Linear regressions were then performed for male participants. For low self-esteem, the prediction model was statistically significant, $[\mathrm{F}(1,71)=15.18, \mathrm{p}<0.001]$, with IA accounting for approximately $17.8 \%$ of the variance of LSE $(\mathrm{R} 2=0.178$, Adjusted R2 $=0.166)$. However, for drive for thinness, the prediction model was not statistically significant $(\mathrm{p}>0.05)$.

\subsubsection{Internet Addiction \& Body-Esteem scores}

Several linear regressions were conducted to assess whether internet addiction was a significant predictor of scores on the Body-esteem Scale. Linear regressions were performed for female participants first. For sexual attractiveness, the prediction model was statistically significant $[\mathrm{F}(1,189)=4.38, \mathrm{p}<0.05]$, with IA accounting for approximately $2.3 \%$ of the variance of LSE $(\mathrm{R} 2=0.023$, Adjusted $\mathrm{R} 2=0.018)$. For physical condition, the prediction model was statistically significant $[\mathrm{F}(1,189)=14.12, \mathrm{p}<0.001]$, with IA accounting for approximately $7 \%$ of the variance of PC $(\mathrm{R} 2=0.070$, Adjusted R2 = 0.065).

Linear regressions were then performed for male participants. For upper body strength, the prediction model was statistically significant $[\mathrm{F}(1,72)=9.66, \mathrm{p}<0.01]$, with IA accounting for approximately $12 \%$ of the variance of PC $(\mathrm{R} 2$ $=0.120$, Adjusted R2 $=0.107)$. However, for physical attractiveness and physical condition, the prediction model was not statistically significant $(\mathrm{p}>0.05)$.

\section{Discussion}

The primary objective of the current study was to evaluate the contribution of internet appearance exposure and internet addiction to predicting levels of body dissatisfaction, the drive for thinness, body-esteem, and self-esteem; within a varied sample in terms of age and gender. As there were significant differences between males and females on reported levels of the EDI-3 measures, the data analysis was conducted separately. Prior research had identified an association between exposure to image-focused media and body dissatisfaction in young women (Tiggemann \& Slater, 2013). However, this connection had not yet been explored in the adult and male population. Parallel with the findings of Tiggemann and Miller, the current study found that internet appearance exposure was significantly associated with higher levels of drive for thinness; suggesting that exposure to the thin-ideal body images presented online appears to reinforce women's desire to achieve the ideal slim female body (Tiggemann \& Miller, 2010). Therefore, further empirical evidence has been found to support that exposure to the unrealistic beauty standards portrayed in the media can contribute to the development of eating disorders such as anorexia-nervosa and bulimia (Spettigue \& Henderson, 2004; Tiggemann, 2003).

In contrast to earlier findings that identified a relationship between image-focused internet use and body dissatisfaction, internet appearance exposure failed to demonstrate a significant association with any other measures of body image in the present sample (Bair, Kelly, Serdar, \& Mazzeo, 2012). A possible explanation for this discrepancy is that the participants may have found it difficult to determine how much time they spent on each individual website. For instance, the amount of time spent online may vary considerably from day to day, meaning averages may have been inaccurate, and many individuals are able to visit different websites at the same time; thus making it difficult to determine individual media dosages (Tiggemann \& Miller, 2010).

Research has found that the reporting of sensitive information in web-based questionnaires can be prone to socially desirable responses, as the social distance and impersonal nature of the internet can inhibit trust development (Joinson, 
2001). Link and Mokdad found a considerable variation in the participants' estimates of heavy drinking when responding to an internet-based survey (Link \& Mokdad, 2005). Block contends a difficulty when attempting to measure internet addiction, as the phenomenon is clouded by shame, denial and minimisation; consequently, individuals engaging in high levels of internet use may have felt unable to disclose their true times spent online, thereby leading to the potential under-reporting of internet consumption (Block, 2008). Future research would benefit from a more sophisticated and objective recording mechanism of internet use, such as tracking the internet sites visited or viewing individual internet history records.

The apparent lack of significant findings can be explained by a possible inadequacy with the procedure for calculating participants' internet appearance exposure score. Three independent raters used a 3-point likert scale $(0=$ no emphasis, $1=$ some emphasis and $2=$ large emphasis) to rate the list of 10 websites on appearance focus, a procedure recommended by Tiggemann and Miller (2010). In practice, however, most rating scales such as the likert-type and attitude measures contain either five or seven response categories (Bearden, Netemeyer, \& Mobley, 1993). It could be argued that the use of a 3-point scale lacks sufficient points of discrimination, meaning that the raters may have been forced to choose the next best alternative; thus introducing the possibility of measurement error. Indeed, psychometric literature has suggested that having more scale points generally leads to improved reliability, with Preston and Colman finding the optimum number of response categories to be seven, with test-retest reliability decreasing for scales with more than 10 and less than four categories (Preston \& Colman, 2000). Future modification of the study would involve asking the raters to use a 7-point likert scale to determine website appearance focus to increase the reliability of the scoring measure. Additionally, the independent raters differed in terms of age and gender, which may impact how individuals view the appearance-focused content of each website.

In regards to the male participants, internet appearance exposure failed to demonstrate a significant association with any of the body image measures. A non-significant association with a drive for thinness was expected, as research has shown that men place more focus on increasing muscle mass rather than losing weight (McCreary \& Sasse, 2000; Petrie, et al., 2010). However, the lack of association with body-esteem measures suggests that other factors identified within the literature search, such as competitiveness (Blashill, 2011), may have greater relevance to the male population than appearance exposure. The items on the body dissatisfaction subscale of the EDI-3 were designed specifically to measure discontentment in women; therefore, the scores generated by the subscale may incorrectly reflect the true levels of body dissatisfaction in the male participants. In a clinical comparison of men and women on subscales of the EDI-3, Stanford and Lemberg found that men scored significantly lower in levels of dissatisfaction with appearance in comparison to women (Stanford \& Lemberg, 2012), which contradicts previous research findings that have shown that in some areas both genders report equal levels of body dissatisfaction (Ricciardelli \& McCabe, 2001). The subscale was incapable of discriminating between men with and men without eating disorders, with the researchers thereby concluding that eating disorders vary significantly amongst genders and that the subscale is incapable of adequately capturing body dissatisfaction as it presents in males. This suggests the need for the development of an eating disorder assessment tool that features body regions typically of concern for males, such as "have larger arms" and "have a 'V' shaped body," in order to produce a more reliable and valid measure of body dissatisfaction in men (Parks \& Read, 1997).

The current study found a significant association between internet addiction and a variety of body image concerns in both males and females. Women who reported higher levels of internet addiction scored significantly lower on measures of perceived sexual attractiveness and physical condition, and demonstrated a higher drive for thinness, body dissatisfaction, and low self-esteem. Male participants who reported high levels of internet addiction scored significantly lower on measures of perceived physical attractiveness, upper body strength, and physical condition, and demonstrated a higher drive for thinness and low self-esteem. These results replicate the previous studies that found an association between internet addiction, self-esteem, and disordered eating amongst both men and women (Koronczai, et al., 2013; Rodgers, et al., 2013). However, the significant association between internet addiction and a drive for thinness was not expected due to men typically placing more focus on increasing muscle mass than losing weight (McCreary \& Sasse, 2000). As internet addiction demonstrated a significant association with the majority of body image variables under investigation, hypothesis two was accepted.

It cannot be determined if these findings indicate that internet addiction contributes to the development of disordered eating and body image concerns, or whether the combination of body concerns and desire to control self-presentation contributes to the development of internet addiction. For instance, Rodgers and colleagues contends that the body satisfaction-problematic internet use relationship may be bidirectional, as individuals experiencing high levels of body image concerns and disordered eating use the internet as a coping mechanism, which in turn can put individuals in a vicious cycle that results in higher levels of psychopathology (Rodgers, et al., 2013). As the present study is correlational in design, causal conclusions between the aforementioned variables cannot be drawn. The question of cause and effect also applies to the relationship between internet appearance exposure and the drive for thinness in 
women, although it is possible that appearance-focused media exposure influences women's drive for the thin-ideal body, it could also be that dissatisfaction with appearance results in women actively seeking internet media that contains pictures of women with the ideal slender body (Tiggemann \& Miller, 2010). Consequently, future research should employ the use of an experimental design in order to ascertain the direction of this relationship.

The results from the regression analysis showed that internet addiction is a significant predictor of the majority of body image variables in both genders. Interestingly, the outcome variable that could most be predicted by internet addiction was low self-esteem in men, accounting for approximately $17.8 \%$ of the variance. A possible explanation for this is provided by Shotton, who explains that introverted males and those with low self-esteem have a greater propensity for internet addiction, as it provides a medium to overcome their deficiencies in social skills, communication, and social relationships (Shotton, 1991).

The drive for thinness was the only body image variable that could be significantly predicted by levels of both internet appearance exposure and internet addiction. Together, the variables explained 3.3\% of the variability in the drive for thinness, suggesting that pathological internet use, in conjunction with exposure to the thin-idealised images depicted online, is capable of producing excessive concerns with dieting and weight in women. However, the low percentage suggests that the model does not explain a high proportion of variability in the response data around the mean. This may reflect the apparent inadequacy of the internet appearance exposure scores, a lack of predictor variables, or that other variables not considered in the present study are better predictors of body image concerns and disordered eating.

For instance, Rodgers et al. (2013) found that BMI, internet addiction symptoms, and body image avoidance explained $54 \%$ of the variability in disordered eating. Wade and Tiggemann (2013) also identified perfectionism as pertinent to levels of body dissatisfaction, accounting for $69 \%$ of the total variance. Fairburn and colleagues identify clinical perfectionism as one of four core mechanisms that contribute to the development and maintenance of eating pathology; thus highlighting the need to examine the contribution of a higher volume of predictor variables to generate a more accurate prediction model (Fairburn, Cooper, \& Shafran, 2003).

The exploration of specific internet and website usage by participants yielded some interesting results. The modal time spent online of four or more hours per day is higher than that ( 2 to 3 hours) reported by Tiggemann and Miller (2010), with social networking sites Facebook and Twitter being reported as the most frequent uses of the internet by both men and women. This reflects the growing popularity of social networking for all ages and genders. Other websites that were frequently reported as been visited by participants were Instagram $(n=7)$, Reddit $(n=9)$ and Tumblr $(n=12)$; these websites are regarded as highly image-focused, allow increased anonymity in comparison to Facebook, and have been implicated in containing material that promotes unhealthy self-destructive behaviours such as self-injury and eating disorders (Lewis \& Arbuthnott, 2014). These findings highlight the increasing prevalence of websites that are providing constant appearance comparison, and therefore, the requirement for further empirical attention in regards to the extent to which the material contained within these websites is fuelling the development of body image concerns.

The study has a number of practical implications. With $94 \%$ of the present sample having regular access to social networking sites and $84 \%$ meeting the criteria for mild internet addiction, this research confirms how much of a hold cyberspace has on society today. As internet addiction, and to a lesser extent internet appearance exposure, were demonstrated to have an association with various body image concerns across a varied participant sample, it highlights the need for pathological internet use to be incorporated into media literacy programs and to be acknowledged as a prolific source of influence on both male and female body image. Individuals of all ages need to be encouraged to take a critical stance towards the unrealistic thin-ideal images presented to them online and to limit their engagement with social networking sites. Furthermore, these findings could be used to advocate changes in website design, so that messages and advertisements that glorify thinness and promote weight loss are discouraged from display.

The present study has made an important contribution to a niche area of research regarding the role of the internet in the formation of body dissatisfaction in the frequently understudied sample of men. Although it has failed to replicate the previous findings that exposure to thin-ideal body images online has a detrimental impact on body satisfaction, it has demonstrated that this form of exposure reinforces women's desire to be thinner in order to achieve the ideal body. Furthermore, it has highlighted the prevalence of pathological internet use in today's society, and the detrimental impact it can have on body image in men and women. Most importantly, it has paved the way for future research to examine the direction of the observed relationships and solidify the aforementioned findings.

\section{References}

Algars, M., Santtila, P., Varjonen, M., Witting, K., Johansson, A., Jern, P., \& Sandnabba, N. K. (2009). The adult body: How age, gender, and body mass index are related to body image. Journal of Aging and Health, 21(8), 1112-1132. http://dx.doi.org/10.1177/0898264309348023 
Aubrey, J. S., \& Taylor, L. D. (2009). The role of lad magazines in priming men's chronic and temporary appearance-related schemata: An investigation of longitudinal and experimental findings. Human Communication Research, 35, 28-58. http://dx.doi.org/10.1111/j.1468-2958.2008.01337.x

Bair, C. E., Kelly, N. R., Serdar, K. L., \& Mazzeo, S. E. (2012). Does the Internet function like magazines? An exploration of image-focused media, eating pathology, and body dissatisfaction. Eating Behaviors, 13(4), 398-401. http://dx.doi.org/10.1016/j.eatbeh.2012.06.003

Bearden, W. O., Netemeyer, R. G., \& Mobley, M. F. (1993). Handbook of marketing scales: Multi item measures for marketing and consumer behavior research. Newbury Park, CA: Sage.

Blashill, A. J. (2011). Gender roles, eating pathology, and body dissatisfaction in men: A meta-analysis. Body Image, 8(1), 1-11. http://dx.doi.org/10.1016/j.bodyim.2010.09.002

Block, J. J. (2008). Issues for DSM-V: Internet Addiction. American Journal of Psychiatry, 165(3), 306-307. http://dx.doi.org/10.1176/appi.ajp.2007.07101556

Blond, A. (2008). Impacts of exposure to images of ideal bodies on male body dissatisfaction: a review. Body Image, 5(3), 244-250. http://dx.doi.org/10.1016/j.bodyim.2008.02.003

Burlew, L. D., \& Shurts, W. M. (2013). Men and Body Image: Current Issues and Counseling Implications. Journal of Counseling \& Development, 91(4), 428-435. http://dx.doi.org/10.1002/j.1556-6676.2013.00114.x

Ceyhan, A. (2008). Predictors of problematic Internet use on Turkish university students. Cyberpsychol Behav, 11(3), 363-366. http://dx.doi.org/10.1089/cpb.2007.0112

Ceyhan, A., Ceyhan, E., \& Kurtyilmaz, Y. (2012). The effect of body image satisfaction on problematic internet use through social support, problem solving skills and depression. The Online Journal of Counselling and Education, 1(3), 84-96.

Chak, K., \& Leung, L. (2004). Shyness and locus of control as predictors of internet addiction and internet use. Cyberpsychol Behav, 7(5), 559-570. http://dx.doi.org/10.1089/cpb.2004.7.559

Clark, L., \& Tiggemann, M. (2006). Appearance Culture in Nine- to 12-Year-Old Girls: Media and Peer Influences on Body Dissatisfaction. Social Development, 15(4), 628-643. http://dx.doi.org/10.1111/j.1467-9507.2006.00361.x

Clausen, L., Rosenvinge, J. H., Friborg, O., \& Rokkedal, K. (2011). Validating the Eating Disorder Inventory-3 (EDI-3): A Comparison Between 561 Female Eating Disorders Patients and 878 Females from the General Population. Journal of Psychopathology and Behavioral Assessment, 33(1), 101-110. http://dx.doi.org/10.1007/s10862-010-9207-4

Dittmar, H. (2009). How Do "Body Perfect" Ideals in the Media Have a Negative Impact on Body Image and Behaviors? Factors and Processes Related to Self and Identity. Journal of Social and Clinical Psychology, 28(1), 1-8. http://dx.doi.org/10.1521/jscp.2009.28.1.1

Dittmar, H., \& Howard, S. (2004). Professional hazards? The impact of models' body size on advertising effectiveness and women's body-focused anxiety in professions that do and do not emphasize the cultural ideal of thinness. $\mathrm{Br} J$ Soc Psychol, 43(Pt 4), 477-497. http://dx.doi.org/10.1348/0144666042565407

Ebizmba. (2013a). Top 15 Most $\quad$ Popular Social Networking Sites. http://wwwebizmbacom/articles/social-networking-websites

Ebizmba. (2013b). Top 15 Most Popular Websites. http://wwwebizmbacom/articles/most-popular-websites

Fairburn, C. G., Cooper, Z., \& Shafran, R. (2003). Cognitive behaviour therapy for eating disorders: a "transdiagnostic" theory and treatment. Behaviour Research and Therapy, 41(5), 509-528. http://dx.doi.org/10.1016/S0005-7967(02)00088-8

Fernandez, S., \& Pritchard, M. (2012). Relationships between self-esteem, media influence and drive for thinness. Eating Behaviors, 13(4), 321-325. http://dx.doi.org/10.1016/j.eatbeh.2012.05.004

Fisher, S. (1964). Sex Differences in Body Perception Psychological Monographs: General and Applied, 78(14), 1-22. http://dx.doi.org/10.1037/h0093871

Franzoi, S. L., \& Shields, S. A. (1984). The Body Esteem Scale: multidimensional structure and sex differences in a college population. J. Pers. Assess., 48(2), 173-178. http://dx.doi.org/10.1207/s15327752jpa4802_12

Garner, D. M. (2004). Eating Disorder Inventory-3.Professional Manual

Grabe, S., Ward, L. M., \& Hyde, J. S. (2008). The role of the media in body image concerns among women: a 
meta-analysis of experimental and correlational studies. Psychol. Bull, 134(3), 460-476. http://dx.doi.org/10.1037/0033-2909.134.3.460

Heinberg, L. J. (1996). Theories of body image disturbance: Perceptual, developmental, and sociocultural factors In J K Thompson (Eds), Body image, eating disorders, and obesity: An integrative guide for assessment and treatment. Washington, DC: American Psychological Association.

Heinberg, L. J., \& Thompson, J. K. (1995). Body Image and Televised Images of Thinness and Attractiveness: A Controlled Laboratory Investigation. Journal of Social and Clinical Psychology, 14(4), 325-338. http://dx.doi.org/10.1521/jscp.1995.14.4.325

Joinson, A. N. (2001). Knowing me, knowing you: reciprocal self-disclosure in Internet-based surveys. Cyberpsychol Behav., 4(5), 587-591. http://dx.doi.org/10.1089/109493101753235179

Knauss, C., Paxton, S. J., \& Alsaker, F. D. (2007). Relationships amongst body dissatisfaction, internalisation of the media body ideal and perceived pressure from media in adolescent girls and boys. Body Image, 4(4), 353-360. http://dx.doi.org/10.1016/j.bodyim.2007.06.007

Koronczai, B., Kokonyei, G., Urban, R., Kun, B., Papay, O., Nagygyorgy, K., . . . Demetrovics, Z. (2013). The mediating effect of self-esteem, depression and anxiety between satisfaction with body appearance and problematic internet use. Am. J. Drug Alcohol Abuse, 39(4), 259-265. http://dx.doi.org/10.3109/00952990.2013.803111

Lewis, S. P., \& Arbuthnott, A. E. (2014). Non-suicidal self-injury, eating disorders, and the internet. L. Claes \& J. J. Muehlenkamp (Eds.), Non-suicidal self-injury in eating disorders: Advancements in etiology and treatment (pp. 273-293). Verlag Berline Heidelberg: Springer. http://dx.doi.org/10.1007/978-3-642-40107-7_16

Link, M. W., \& Mokdad, A. H. (2005). Effects of survey mode on self-reports of adult alcohol consumption: a comparison of mail, web and telephone approaches. J. Stud. Alcohol, 66(2), 239-245. http://dx.doi.org/10.15288/jsa.2005.66.239

Marshall, C., Lengyel, C., \& Utioh, A. (2012). Body dissatisfaction among middle-aged and older women. Can J. Diet Pract Res., 73(2), e241-e247. http://dx.doi.org/10.3148/73.2.2012.e241

McCreary, D. R., \& Sasse, D. K. (2000). An exploration of the drive for muscularity in adolescent boys and girls. J. Am. Coll. Health, 48(6), 297-304. http://dx.doi.org/10.1080/07448480009596271

Morris, A. M., \& Katzman, D. K. (2003). The impact of the media on eating disorders in children and adolescents. Paediatrics \& Child Health, 8(5), 287-289.

ONS. (2012). Social Networking: The UK as a Leader in Europe.

Parks, P. S., \& Read, M. H. (1997). Adolescent male athletes: body image, diet, and exercise. Adolescence, 32(127), 593-602.

Petrie, T., Greenleaf, C., \& Martin, S. (2010). Biopsychosocial and Physical Correlates of Middle School Boys' and Girls’ Body Satisfaction. Sex Roles, 63(9-10), 631-644. http://dx.doi.org/10.1007/s11199-010-9872-5

Preston, C. C., \& Colman, A. M. (2000). Optimal number of response categories in rating scales: reliability, validity, discriminating power, and respondent preferences. Acta. Psychol., 104(1), 1-15. http://dx.doi.org/10.1016/S0001-6918(99)00050-5

Rasmussen, F., Eriksson, M., \& Nordquist, T. (2007). Bias in height and weight reported by Swedish adolescents and relations to body dissatisfaction: the COMPASS study. Eur. J. Clin. Nutr, 61(7), 870-876. http://dx.doi.org/10.1038/sj.ejcn.1602595

Ricciardelli, L. A., \& McCabe, M. P. (2001). Dietary restraint and negative affect as mediators of body dissatisfaction and bulimic behavior in adolescent girls and boys. Behav. Res. Ther, 39(11), 1317-1328. http://dx.doi.org/10.1016/S0005-7967(00)00097-8

Rodgers, R. F., Melioli, T., Laconi, S., Bui, E., \& Chabrol, H. (2013). Internet addiction symptoms, disordered eating, and body image avoidance. Cyberpsychol Behav. Soc. Netw., 16(1), 56-60. http://dx.doi.org/10.1089/cyber.2012.1570

Rodin, J., Silberstein, L., \& Striegel-Moore, R. (1984). Women and weight: a normative discontent. Nebr. Symp. Motiv., 32, 267-307.

Rukavina, T., \& Pokrajac-Bulian, A. (2006). Thin-ideal internalization, body dissatisfaction and symptoms of eating disorders in Croatian adolescent girls. Eat Weight Disord, 11(1), 31-37. http://dx.doi.org/10.1007/BF03327741

Secord, P. F., \& Jourard, S. M. (1953). The appraisal of body-cathexis: body-cathexis and the self. J. Consult Psychol., 
17(5), 343-347. http://dx.doi.org/10.1037/h0060689

Shotton, M. (1991). The costs and benefits of computer addiction. Behaviour and Information Technology, 10, $219-230$. http://dx.doi.org/10.1080/01449299108924284

Siomos, K., \& Angelopoulos, N. (2008). Internet addiction disorder. Psychiatrike 19(1), 52-58.

Smolak, L., \& Stein, J. A. (2006). The relationship of drive for muscularity to sociocultural factors, self-esteem, physical attributes gender role, and social comparison in middle school boys. Body Image, 3(2), 121-129. http://dx.doi.org/10.1016/j.bodyim.2006.03.002

Song, I., LaRose, R., Eastin, M. S., \& Lin, C. A. (2004). Internet gratifications and internet addiction: on the uses and abuses of new media. Cyberpsychol Behav., 7(4), 384-394. http://dx.doi.org/10.1089/cpb.2004.7.384

Spettigue, W., \& Henderson, K. (2004). Eating Disorders and the Role of the Media. The Canadian child and adolescent psychiatry review, 13(1), 16-19.

Stanford, S. C., \& Lemberg, R. (2012). A clinical comparison of men and women on the eating disorder inventory-3 (EDI-3) and the eating disorder assessment for men (EDAM). Eat Disord, 20(5), 379-394. http://dx.doi.org/10.1080/10640266.2012.715516

Subrahmanyam, K., \& Greenfield, P. (2008). Online communication and adolescent relationships. Future Child, 18(1), 119-146. http://dx.doi.org/10.1353/foc.0.0006

Suler, J. (2004). The online disinhibition effect. Cyberpsychol Behav., 7(3), 321-326. http://dx.doi.org/10.1089/1094931041291295

Tabachnick, B. G., \& Fidell, L. S. (1989). Using multivariate statistics (2nd ed). New York: HarperCollins.

Tiggemann, M. (2003). Media exposure, body dissatisfaction and disordered eating: television and magazines are not the same! European Eating Disorders Review, 11(5), 418-430. http://dx.doi.org/10.1002/erv.502

Tiggemann, M., \& Lynch, J. E. (2001). Body image across the life span in adult women: the role of self-objectification. Dev. Psychol., 37(2), 243-253. http://dx.doi.org/10.1037/0012-1649.37.2.243

Tiggemann, M., \& Miller, J. (2010). The Internet and Adolescent Girls' Weight Satisfaction and Drive for Thinness. Sex Roles, 63(1-2), 79-90. http://dx.doi.org/10.1007/s11199-010-9789-z

Tiggemann, M., \& Slater, A. (2013). NetGirls: The Internet, Facebook, and body image concern in adolescent girls. International Journal of Eating Disorders, 46(6), 630-633. http://dx.doi.org/10.1002/eat.22141

Wade, T. D., \& Tiggemann, M. (2013). The role of perfectionism in body dissatisfaction. Journal of Eating Disorders, 1, 2-2. http://dx.doi.org/10.1186/2050-2974-1-2

Weinstein, A., \& Lejoyeux, M. (2010). Internet addiction or excessive internet use. Am J Drug Alcohol Abuse, 36(5), 277-283. http://dx.doi.org/10.3109/00952990.2010.491880

Yang, S. C., \& Tung, C.-J. (2007). Comparison of Internet addicts and non-addicts in Taiwanese high school. Computers in Human Behavior, 23(1), 79-96. http://dx.doi.org/10.1016/j.chb.2004.03.037

Young, K. S. (1998a). Internet Addiction Test (IAT). http://www.netaddiction.com/resources/internet_addiction_test.htm

Young, K. S. (1998b). Internet Addiction: The Emergence of a New Clinical Disorder. CyberPsychology \& Behavior, 1(3), 237-244. http://dx.doi.org/10.1089/cpb.1998.1.237

\section{(cc) $\mathrm{BY}$}

This work is licensed under a Creative Commons Attribution 3.0 License. 